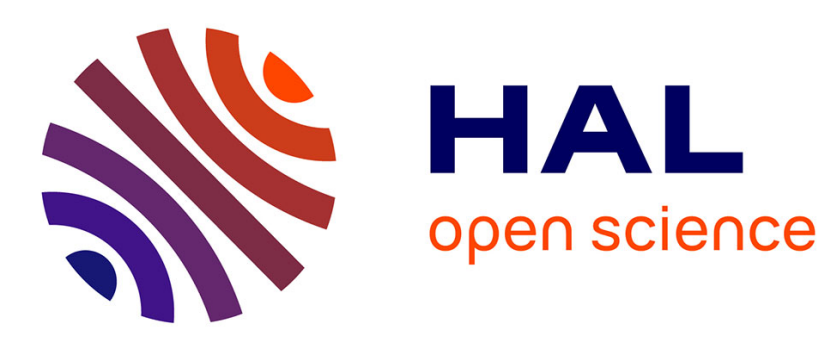

\title{
Modélisation analytique des machines à aimants à induit sans encoches
}

\author{
Bertrand Nogarède, Michel Lajoie-Mazenc, Bernard Davat
}

\section{To cite this version:}

Bertrand Nogarède, Michel Lajoie-Mazenc, Bernard Davat. Modélisation analytique des machines à aimants à induit sans encoches. Revue de Physique Appliquée, 1990, 25 (7), pp.707-720. 10.1051/rphysap:01990002507070700 . jpa-00246233

\section{HAL Id: jpa-00246233 https://hal.science/jpa-00246233}

Submitted on 1 Jan 1990

HAL is a multi-disciplinary open access archive for the deposit and dissemination of scientific research documents, whether they are published or not. The documents may come from teaching and research institutions in France or abroad, or from public or private research centers.
L'archive ouverte pluridisciplinaire HAL, est destinée au dépôt et à la diffusion de documents scientifiques de niveau recherche, publiés ou non, émanant des établissements d'enseignement et de recherche français ou étrangers, des laboratoires publics ou privés. 
Classification

Physics Abstracts

$02.70-41.10-41.90-75.90$

\title{
Modélisation analytique des machines à aimants à induit sans encoches
}

\author{
Bertrand Nogarede $\left({ }^{1}\right)$, Michel Lajoie-Mazenc $\left({ }^{1}\right)$ et Bernard Davat $\left({ }^{2}\right)$ \\ (') Laboratoire d'Electrotechnique et d'Electronique Industrielle, Unité de Recherche Associée au CNRS \\ $\mathrm{n}^{\circ}$ 847, ENSEEIHT, 31071 Toulouse, France \\ (2) Initialement LEEI, actuellement Groupe de Recherche en Electrotechnique et Electronique de Nancy, \\ France
}

(Reçu le 5 octobre 1989, révisé le 25 janvier 1990, accepté le 22 février 1990)

\begin{abstract}
Résumé. - Dans cet article une méthode d'étude adaptée aux machines à aimants à induit sans encoches est proposée. Cette méthode est basée sur la résolution analytique de l'équation du champ magnétique dans les différentes zones constitutives de la machine. Après avoir présenté les bases de la méthode, les auteurs insistent sur les particularités relatives à la description mathématique de l'inducteur à aimants et de l'enroulement d'induit. La procédure de résolution des équations est ensuite explicitée et le résultat est utilisé pour calculer les principales grandeurs caractéristiques de la machine : induction, f.é.m., couple. Les résultats théoriques sont finalement confrontés aux mesures effectuées sur un prototype particulier.
\end{abstract}

\begin{abstract}
A method suitable for the study of slotless permanent magnet machines is proposed. This method is based on an analytical solution of the field equation in the different parts of the machine. After a presentation of the basis of the method, the authors insist on the pecularities relating to the mathematical description of the magnet inductor and the slotless armature winding. The solving process is then explained and the result is used to calculate main characteristics of the machine : field density, e.m.f., torque. Finally, the theoretical results are compared to the tests realized on a special prototype.
\end{abstract}

Introduction.

Depuis quelques années, les machines à induit sans encoches, dont la structure de bobinage fut utilisée couramment jusqu'au début du siècle font l'objet d'un regain d'intérêt. Un certain nombre d'études ont tout d'abord souligné les avantages de cette technologie dans le cadre de machines de fortes puissances, e es que ur o-a ernateurs, , et moteurs de traction $[4,5]$.

D'autre part, cette structure d'induit a été plus récemment mise en œuvre dans le cas de machines synchrones à aimants permanents en vue notamment de réduire les ondulations de couple [6,7].

Nous présentons, dans cet article, une méthode d'étude de ces machines, dont la structure relativement simple, schématisée sur la figure 1 , se prête bien à un calcul analytique du champ (en deux dimensions) $[8,9]$.

Nous exposons dans un premier temps les bases de ce calcul: après en avoir présenté les principes généraux, nous envisageons la modélisation des sources du champ, correspondant aux aimants et aux courants.

Nous établissons dans une deuxième partie les expressions du potentiel vecteur dans les différentes zones du domaine d'étude.

Après avoir déterminé les expressions des grandeurs caractérisant le fonctionnement de la machine, nous procédons enfin à la validation de ce calcul, en confrontant les résultats obtenus avec ceux issus de l'expérience.

\section{Bases de la modélisation.}

1.1 PrincIPE. - Le calcul analytique, en deux dimensions, que nous présentons ici s'applique aux structures électromagnétiques susceptibles d'être subdivisées en un certain nombre de zones concentriques, d'épaisseur et de perméabilité constantes, dans lesquelles sont disposés les matériaux magnéti- 


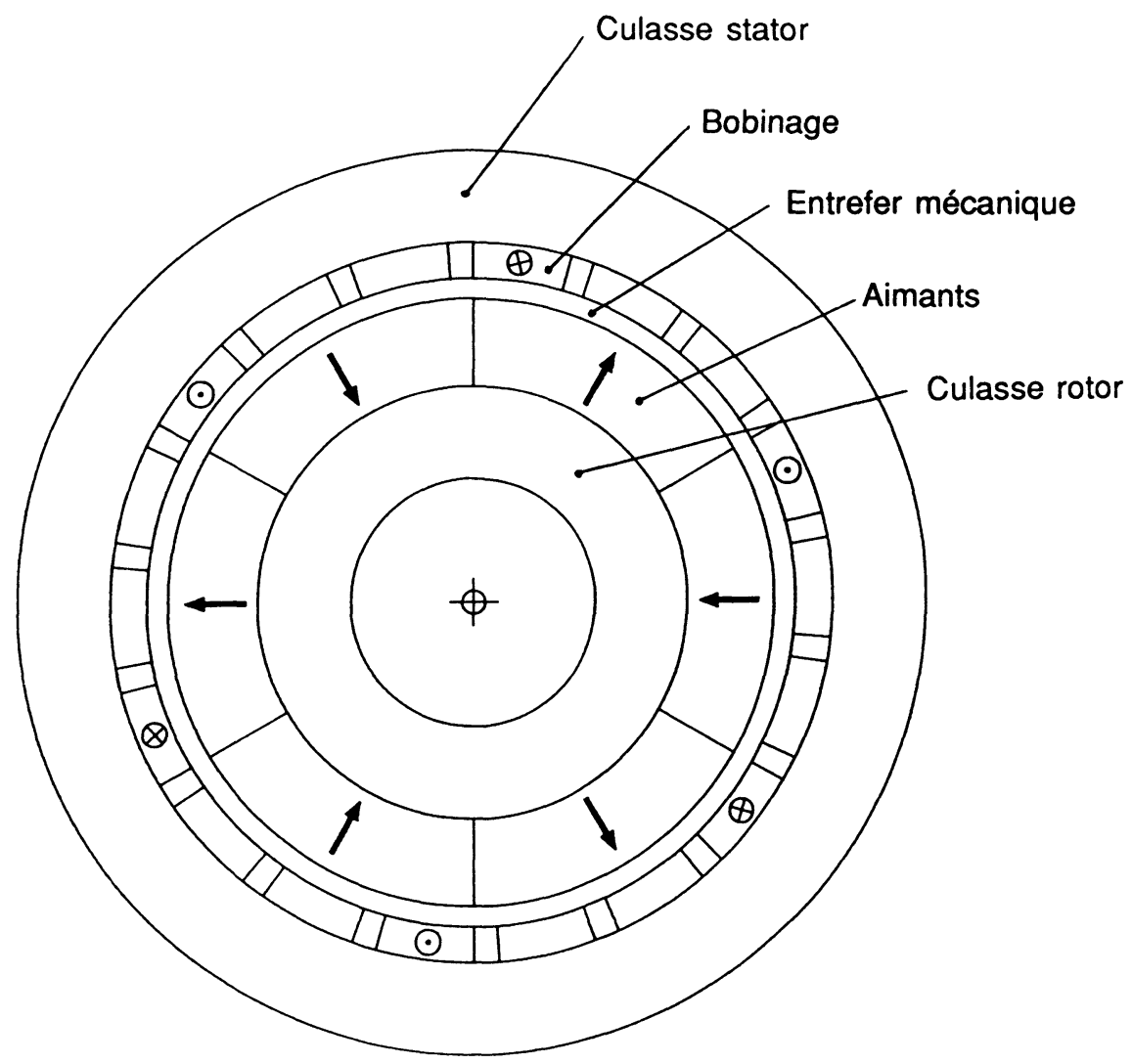

Fig. 1. - Structure d'une machine à aimants sans encoches.

[Structure of a slotless permanent magnet machine.]

ques et où interviennent les sources du champ, aimants ou courants.

La machine sans encoches à pôles lisses, schématisée sur la figure 1 , répond à cette définition. En effet, elle ne fait pas intervenir de matériau magnétique au niveau du bobinage de l'induit et, dans la mesure où les différents pôles sont jointifs, la couronne aimantéc constituant l'inducteur possède une perméabilité constante égale à la perméabilité de recul des aimants $\mu_{\mathrm{p}}$.

Lorsque les pôles de l'inducteur sont réalisés au moyen de blocs non jointifs, séparés par des secteurs amagnétiques, la modélisation proposée est encore applicable à condition toutefois de supposer que la perméabilité des aimants est égale à $\mu_{0}$. Ce n'est pas une hypothèse très contraignante puisque $\mu_{\mathrm{p}}$ est très voisin de $\mu_{0}$ (généralement $\mu_{\mathrm{p}} \simeq 1,1 \mu_{0}$ ).

Bien qu'il soit tout à fait possible de prendre en compte des culasses de perméabilité de valeur constante et finie, elles sont, dans ce qui suit, supposées de perméabilité infinie afin de simplifier la présentation de la modélisation en diminuant de deux le nombre de zones à prendre en considération.

Dans le même esprit, le bobinage stator n'est

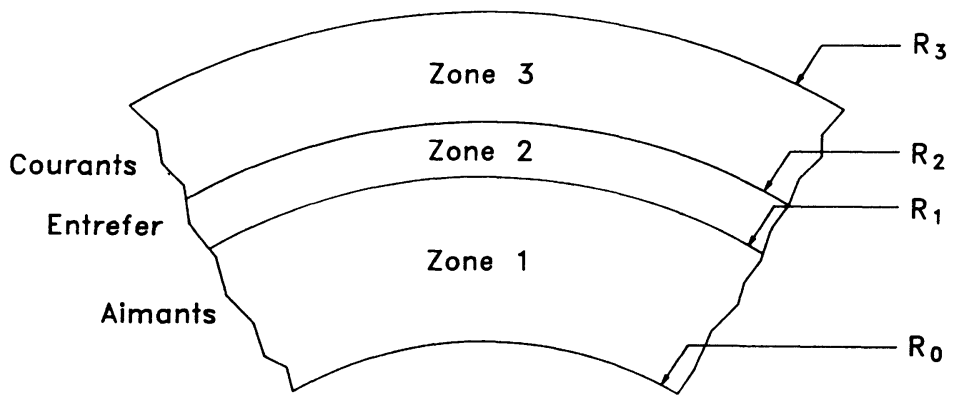

Fig. 2. - Domaine d'étude.

[Area under study.] 
décrit ici que par une seule zone concentrique, bien qu'il soit tout à fait envisageable d'en multiplier le nombre, afin notamment de prendre en compte des bobinages comportant plusieurs couches. Dans ces conditions, le domaine d'étude se réduit à 3 zones élémentaires, représentées sur la figure 2, correspondant respectivement aux aimants du rotor (entre les cercles de rayon $R_{0}$ et $R_{1}$ ), à l'entrefer mécanique (entre les cercles de rayon $R_{1}$ et $R_{2}$ ) et au bobinage stator (entre les cercles de rayon $R_{2}$ et $R_{3}$ ).

Dans l'approximation des états quasi stationnaires, la modélisation de la machine peut être faite en utilisant les deux équations :

$$
\operatorname{rot} \mathbf{H}=\mathbf{J} ; \quad \mathbf{B}=\operatorname{rot} \mathbf{A}
$$

où $\mathbf{H}$ et $\mathbf{B}$ désignent le champ et l'induction magnétique, $\mathbf{J}$ la densité volumique de courant et $\mathbf{A}$ le potentiel vecteur.

L'induction $\mathbf{B}$ est reliée au champ $\mathbf{H}$, en dehors des aimants, par la relation :

$$
\mathbf{B}=\mu_{0} \mathbf{H} .
$$

Les aimants magnétiquement rigides, actuellement employés dans les machines électriques, possèdent une caractéristique de désaimantation linéaire dans la zone normale d'utilisation. Ils peuvent donc être représentés par l'équation :

$$
\mathbf{B}=\mu_{\mathrm{p}} \mathbf{H}+\mathbf{M}
$$

où $\mathbf{M}$ désigne l'induction rémanente qui est égale à l'aimantation à champ nul et que nous appellerons, pour simplifier dans ce qui suit, "aimantation ».

La forme générale de l'équation à résoudre est donc :

$$
\operatorname{rot}\left(\frac{\operatorname{rot} \mathbf{A}}{\mu}\right)=\mathbf{J}+\operatorname{rot}\left(\frac{\mathbf{M}}{\mu}\right)
$$

où $\mu$ désigne, de manière générale, la perméabilité ( $\mu=\mu_{0}$ dans l'air et les conducteurs, $\mu=\mu_{\mathrm{p}}$ dans les aimants permanents).

Pour appliquer cette équation à l'étude de la machine représentée sur la figure 1 , on utilise un système de coordonnées cylindriques dont l'axe $\mathrm{O} z$ est dirigé suivant l'axe de la machine (perpendiculaire au lan de la Fi .. De lus si on su ose, pour travailler en deux dimensions, que le système électromagnétique est invariant par translation suivant cet axe $\mathrm{O} z$, la densité de courant $\mathbf{J}$ et le potentiel vecteur A n'ont qu'une composante suivant $\mathrm{O} z$ que nous notons pour simplifier, $A$ et $J$.

Dans ces conditions, et en tenant compte du fait que les perméabilités des différentes zones de la figure 2 sont constantes ( $\mu=\mu_{0}$ ou éventuellement $\mu_{\mathrm{p}}$ pour les aimants), l'équation (1) s'écrit :

$\frac{\partial^{2} A}{\partial r^{2}}+\frac{1}{r} \frac{\partial A}{\partial r}+\frac{1}{r^{2}} \frac{\partial^{2} A}{\partial \theta^{2}}=$

$$
=\frac{1}{r} \frac{\partial M_{r}}{\partial \theta}-\frac{1}{r} \frac{\partial}{\partial r}\left(r M_{\theta}\right)-\mu J .
$$

$M_{r}$ et $M_{\theta}$ représentent respectivement les composantes radiale et tangentielle de l'aimantation.

Si on néglige les phénomènes liés aux courants de Foucault dans les courants et dans les aimants et si on considère que les courants agissant dans le système sont connus, $J$ désigne, dans cette équation, la densité de courant imposée dans le bobinage par le circuit extérieur.

Le principe du calcul consiste à rechercher l'expression du potentiel vecteur satisfaisant à l'équation (2) dans chacune des zones du domaine représentées sur la figure 2 .

Dans chacune des zones, le second membre de l'équation (2) se réduit au terme correspondant au type de source présent dans la zone.

Après avoir déterminé les expressions de ces termes, fonctions de la variable angulaire $\theta$ et du temps, on peut obtenir, dans chaque zone, la solution générale de l'équation (2). Les constantes intervenant dans ces expressions sont ensuite identifiées à l'aide des conditions aux limites définissant les différentes zones.

1.2 Modélisation Des SourCES. - Compte tenu de la périodicité de répartition des sources sur le pourtour de la machine, les termes correspondants peuvent s'exprimer sous forme de séries de Fourier, sur la variable $\boldsymbol{\theta}$.

1.2.1 Expression du terme source dî aux courants. - Afin de déterminer la densité de courant $J$ en tout point de la zone correspondante (zone 3), il faut dans un premier temps décrire la répartition des conducteurs à l'intérieur de ce domaine. Pour ce faire, nous introduisons ici la grandeur $C$, dont la valeur absolue définit, en tout point du bobinage, la densité surfacique de conducteurs. Le signe de $C$ permet quant à lui de prendre en compte le fait qu'il existe dans le bobinage des conducteurs " aller » et des conducteurs " retour".

Nous supposons que le bobinage $m$ phasé de la machine est parfaitement symétrique, c'est-à-dire que la répartition des conducteurs d'une phase se déduit de celle e a p ase voisıne par une sımp e rotation d'angle électrique $\frac{2 \pi}{m}$. Si $C_{n}$ représente la densité des conducteurs, de la phase $n$, on a ainsi :

$$
C_{n}(\theta)=C_{1}\left(\theta-\frac{n-1}{p} \frac{2 \pi}{m}\right)
$$

où $p$ désigne le nombre de paires de pôles de la machine.

Il suffit donc, pour définir l'ensemble du bobinage, de déterminer l'expression de la densité de conducteurs de la phase 1 . 
Axe de symétrie

du groupement "aller"

Axe de symétrie de la phase,
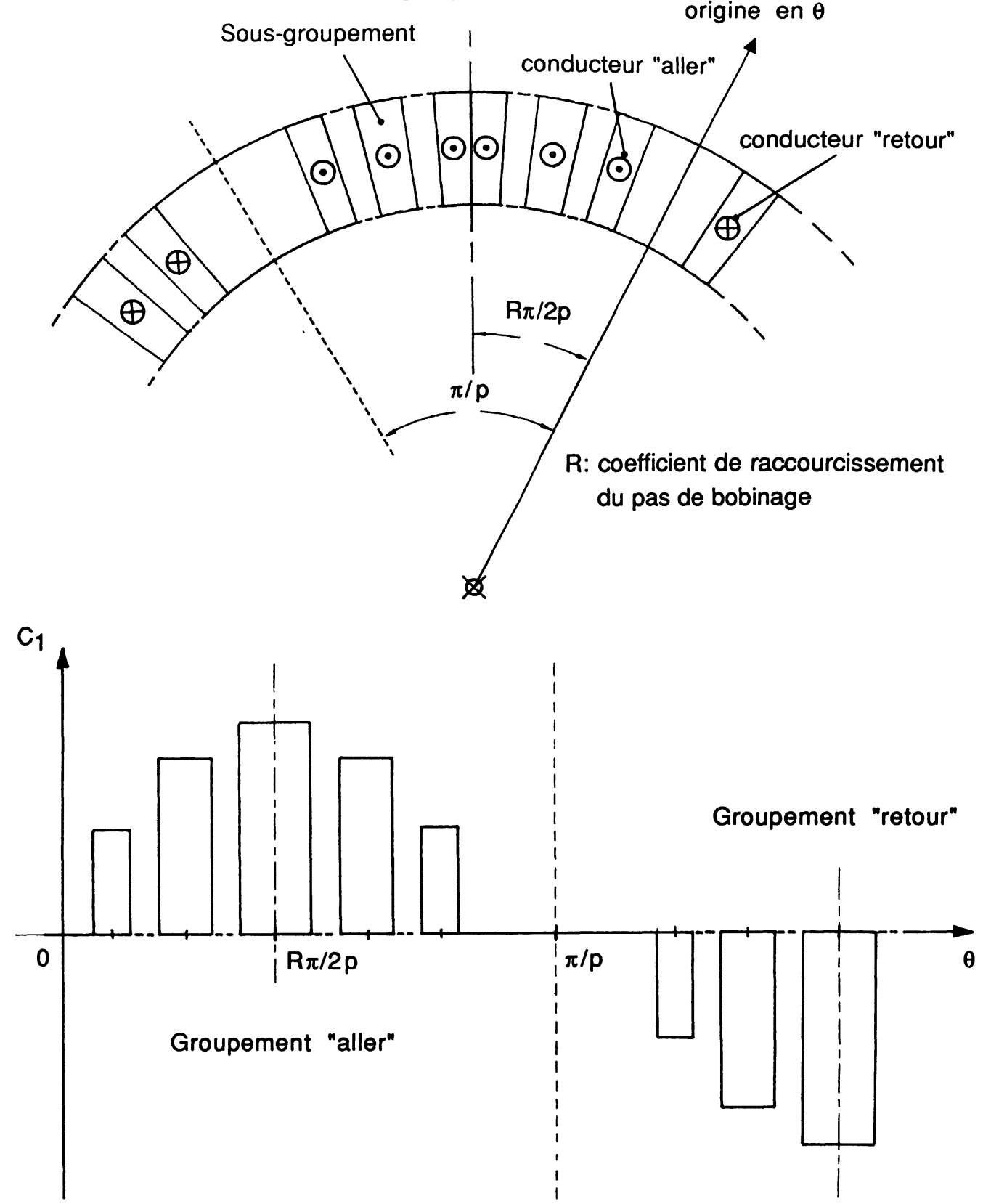

Fig. 3. - Répartition des conducteurs de la phase 1 et densité de conducteurs correspondante.

[Distribution of the conductors of phase 1 and corresponding conductor density.]

Afin de prendre en compte des bobinages relativement complexes, comme on peut le voir sur la figure 3, nous supposons d'une manière générale que les conducteurs de chaque phase se répartissent le long de la couche d'induit, en un certain nombre de sous-groupements. Ainsi, la modélisation permet d'envisager en particulier le cas des bobinages à pas raccourci.

Sur cette figure 3 est également représentée la densité de conducteurs correspondant à cette répartition.
L'origine en $\theta$ étant confondue avec l'axe de la phase 1 , et compte tenu des symétries, la densité $C_{1}$ peut alors s'écrire:

$$
C_{1}(\theta)=\sum_{g=1}^{+\infty} S_{g} \sin g p \theta
$$

Les coefficients de Fourier $S_{g}$ sont calculés à partir des caractéristiques dimensionnelles du bobinage.

A partir de cette définition, il faut déterminer l'expression de la densité de courant $J$ en un point de 
l'induit, en faisant intervenir les courants alimentant ce que l'on peut écrire sous forme complexe : chaque phase.

Si $I_{n}(t)$ désigne le courant parcourant les conducteurs de la phase $n$ à l'instant $t$, on a :

$$
J(\theta, t)=\sum_{g=-\infty}^{+\infty} \gamma_{g} \mathrm{e}^{j g p \theta}
$$

$$
\begin{aligned}
J(\theta, t) & =\sum_{n=1}^{m} I_{n}(t) \cdot C_{n}(\theta)= & \text { avec : } & \\
& =\sum_{n=1}^{m} I_{n}(t) \cdot \sum_{g=1}^{+\infty} S_{g} \sin g\left[p \theta-(n-1) \frac{2 \pi}{m}\right] & & g_{g}=\frac{S_{g}}{2 j} \sum_{n=1}^{m} I_{n}(t) \mathrm{e}^{-j g(n-1) \frac{2 \pi}{m}}
\end{aligned}
$$

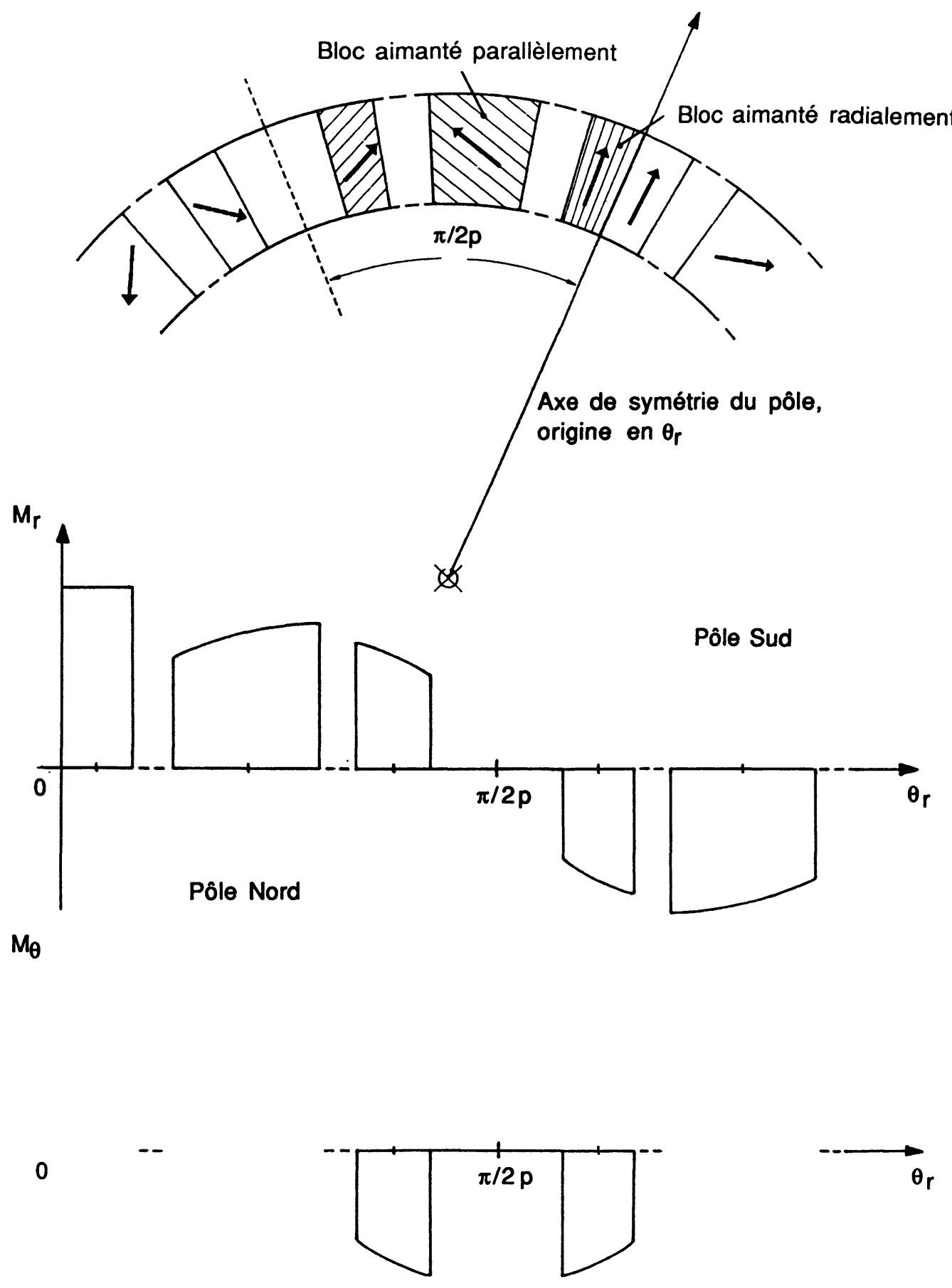

Fig. 4. - Répartition de l'aimantation sur le rotor.

[Distribution of magnetic polarisation on the rotor.] 
La densité de courant $J$ étant réelle, on a :

$$
\mathfrak{y}_{-g}=\mathfrak{J}_{g}^{*} .
$$

Les coefficients $S_{g}$ doivent donc satisfaire à la condition :

$$
S_{-g}=-S_{g} .
$$

Remarquons que cette modélisation fait intervenir de façon explicite l'épaisseur du bobinage. Elle permettra donc de rendre compte de l'évolution des phénomènes dans cette épaisseur, ce qui ne serait pas possible avec une représentation du bobinage par une simple densité linéique.

1.2.2 Expressions des termes sources dûs aux aimants. - La prise en compte des aimants dans les modélisations analytiques des machines électriques se fait très souvent par le biais de courants superficiels équivalents [8-11]. Toutefois, cette analogie n'est pas obligatoire [12], dans la mesure où l'aimantation apparaît directement dans l'équation (2).

De la même manière que pour le terme source courant, il s'agit donc de déterminer les séries de Fourier correspondant à la répartition des aimantations radiale et tangentielle dans la zone des aimants.

Le rotor de la machine met en œuvre $2 p$ pôles identiques, symétriques par rapport à leur axe, euxmêmes éventuellement constitués d'un certain nombre de blocs aimantés.

En ce qui concerne l'aimantation, nous envisageons ici seulement les cas suivants :

L'aimantation a une intensité constante à l'intérieur de chaque bloc élémentaire et elle peut être soit radiale, soit parallèle à une direction quelconque donnée.

La structure générale d'un pôle illustrant ces diverses possibilités ainsi que les composantes $M_{r}$ et $M_{\theta}$ de l'aimantation correspondante sont données sur la figure 4.

Les caractéristiques du pôle étant ainsi définies, on peut décomposer en série les aimantations $M_{r}$ et $M_{\theta}$ dans un référentiel lié au rotor.

$\theta_{r}$ représentant l'angle de repérage dans ce référentiel, il vient, compte tenu de l'origine en $\theta_{r}$ choisie et des différentes symétries :

$$
\begin{aligned}
& M_{r}\left(\theta_{r}\right)=\sum_{g=1}^{+\infty} M_{r_{g}} \cos \left(g p \theta_{r}\right), \\
& M_{\theta}\left(\theta_{r}\right)=\sum_{g=1}^{+\infty} M_{\theta_{g}} \sin \left(g p \theta_{r}\right)
\end{aligned}
$$

avec $M_{r_{g}}=M_{\theta_{g}}=0$ pour tout $g$ pair.

La dernière étape consiste enfin à exprimer ces grandeurs dans le référentiel stator, ce qui permet de prendre en compte le mouvement du rotor par rapport au stator.

Si $\theta_{0}$ représente le déphasage, à l'instant $t=0$, entre l'axe de la phase 1 prise comme origine au stator et l'axe origine du rotor, la correspondance entre les coordonnées stator $\theta$ et rotor $\theta_{r}$ est donnée par la relation :

$$
\theta=\theta_{r}+\theta_{0}+\Omega \cdot t
$$

où $\Omega$ désigne la vitesse de rotation mécanique du rotor.

On obtient alors aisément les expressions des aimantations radiale et tangentielle dans le référentiel stator, écrites ici sous forme complexe :

$$
\begin{aligned}
& M_{r}(\theta)=\sum_{g=-\infty}^{+\infty} \mathcal{M}_{r_{g}} \mathrm{e}^{j g p \theta}, \\
& M_{\theta}(\theta)=\sum_{g=-\infty}^{+\infty} \mathcal{M}_{\theta_{g}} \mathrm{e}^{j g p \theta}
\end{aligned}
$$

où :

$$
\begin{aligned}
\mathcal{M}_{r_{g}} & =\frac{M_{r_{g}}}{2} \mathrm{e}^{-j g p \theta_{0}-j g p \Omega t}, \\
\mathcal{M}_{\theta_{g}} & =\frac{M_{\theta_{g}}}{2 j} \mathrm{e}^{-j g p \theta_{0}-j g p \Omega t}
\end{aligned}
$$

avec les conditions :

$$
M_{r_{-g}}=M_{r_{g}}, \quad M_{\theta_{-g}}=-M_{\theta_{g}}
$$

qui entraînent :

$$
\mathcal{M}_{r_{-g}}=\mathcal{M}_{r_{g}}^{*}, \quad \mathcal{M}_{\theta_{-g}}=\mathcal{M}_{\theta_{g}}^{*} .
$$

Les coefficients de Fourier $M_{r_{g}}$ et $M_{\theta_{g}}$ sont calculés à partir des caractéristiques dimensionnelles de l'inducteur.

\section{Calcul du potentiel vecteur.}

Après avoir déterminé les expressions mathématiques des termes sources relatifs aux aimants et aux courants, apparaissant dans l'équation (2), il est désormais possible d'obtenir, dans chaque zone du domaine, la solution de cette équation.

\subsection{EXPRESSIONS DU POTENTIEL VECTEUR.}

Zones de l'entrefer mécanique (Zone 2, Fig. 2). L'équation (1) s'écrit dans cette zone:

$$
\frac{\partial^{2} A_{2}}{\partial r^{2}}+\frac{1}{r} \frac{\partial A_{2}}{\partial r}+\frac{1}{r^{2}} \frac{\partial^{2} A_{2}}{\partial \theta^{2}}=0 .
$$

La solution de cette équation va contenir les différents harmoniques en $\theta$ contenus dans les sources du champ.

On obtient alors l'expression générale suivante :

$$
A_{2}=\sum_{g=-\infty}^{+\infty}\left(E_{g} r^{|g| p}+F_{g} r^{-|g| p}\right) \mathrm{e}^{j g p \theta}
$$


avec, le potentiel vecteur étant réel :

$$
\left\{\begin{array}{l}
E_{-g}=E_{g}^{*} \\
F_{-g}=F_{g}^{*} .
\end{array}\right.
$$

Zone des aimants (Zone 1, Fig. 2). - Avec les hypothèses adoptées au paragraphe 1.2.2 relatives à la répartition de l'aimantation, le terme $\frac{\partial M_{\theta}}{\partial r}$ est nul.

L'équation (1) devient alors dans cette zone :

$$
r \frac{\partial^{2} A_{1}}{\partial r^{2}}+\frac{\partial A_{1}}{\partial r}+\frac{1}{r} \frac{\partial^{2} A_{1}}{\partial \theta^{2}}=\frac{\partial M_{r}}{\partial \theta}-M_{\theta} .
$$

Les expressions des termes $M_{r}$ et $M_{\theta}$ sont données par les relations (4).

La solution s'écrit alors comme la somme de la solution générale de l'équation sans second membre, obtenue dans la zone de l'entrefer, et d'une solution particulière de l'équation avec second membre. Il vient alors :

$A_{1}=\sum_{g=-\infty}^{+\infty}\left(C_{g} r^{|g| p}+D_{g} r^{-|g| p}+\alpha_{g}(r)\right) \mathrm{e}^{j g p \theta}$

avec :

$$
\left\{\begin{array}{l}
\alpha_{g}(r)=\frac{j g p \mathcal{M}_{r_{g}}-\mathcal{M}_{\theta_{g}}}{1-g^{2} p^{2}} \cdot r \text { si } g^{2} p^{2} \neq 1 \\
\alpha_{g}(r)=\frac{1}{2}\left(j g p \mathcal{M}_{r_{g}}-\mathcal{M}_{\theta_{g}}\right) r \log r \text { si } g^{2} p^{2}=1
\end{array}\right.
$$

et : $\left\{\begin{array}{l}C_{-g}=C_{g}^{*} \\ D_{-g}=D_{g}^{*}\end{array}\right.$ puisque le potentiel vecteur est réel.

Zone des courants (Zone 3, Fig. 2). - Le potentiel vecteur vérifie dans cette zone l'équation:

$$
\frac{\partial^{2} A_{3}}{\partial r^{2}}+\frac{1}{r} \frac{\partial A_{3}}{\partial r}+\frac{1}{r^{2}} \frac{\partial^{2} A_{3}}{\partial \theta^{2}}=-\mu_{0} J
$$

associée à l'expression (3) du terme source courant, ce qui conduit à la solution :

$$
A_{3}={ }_{g=-\infty}^{+\infty}\left(G \quad r^{|g| p}+H r^{-|g| p}+\beta(r)\right) \mathrm{e}^{j g p \theta}
$$

avec :

$$
\left\{\begin{array}{l}
\beta_{g}(r)=-\frac{\mu_{0} g_{g}}{4-g^{2} p^{2}} \cdot r^{2} \text { si } g^{2} p^{2} \neq 4 \\
\beta_{g}(r)=-\frac{1}{4} \mu_{0} \xi_{g} r^{2} \log r \quad \text { si } g^{2} p^{2}=4
\end{array}\right.
$$

et : $\left\{\begin{array}{l}G_{-g}=G_{g}^{*} \\ H_{-g}=H_{g}^{*}\end{array}\right.$ puisque le potentiel vecteur est réel.
2.2 Conditions AuX limites. - Les expressions du potentiel vecteur (5), (6) et (7) obtenues au cours du paragraphe précédent font intervenir 6 constantes $C_{g}, D_{g}, E_{g}, F_{g}, G_{g}, H_{g}$. Leur détermination se fait à l'aide des relations supplémentaires qui traduisent les conditions aux limites des différentes zones. Ces conditions, qui correspondent aux conservations de la composante normale de l'induction $B$ d'une part, et de la composante tangentielle du champ magnétique $H$, en l'absence de densité superficielle de courant réelle, d'autre part, s'écrivent en terme de potentiel vecteur sous la forme suivante :

- Conditions aux limites externes du domaine. Les culasses étant de perméabilité infinie, on peut écrire :

$$
\left(\frac{\partial A_{1}}{\partial r}\right)_{r=R_{0}}+M_{\theta}=0, \quad\left(\frac{\partial A_{3}}{\partial r}\right)_{r=R_{3}}=0
$$

- Conditions entre les différentes zones repérées par l'indice $i \in\{1,2\}$.

$$
\left(\frac{1}{r} \frac{\partial A_{i}}{\partial \theta}\right)_{r=R_{i}}=\left(\frac{1}{r} \frac{\partial A_{i+1}}{\partial \theta}\right)_{r=R_{i}}
$$

$$
\begin{aligned}
\frac{1}{\mu_{i}}\left[\left(\frac{\partial A_{i}}{\partial r}\right)_{r=R_{i}}\right. & \left.+M_{\theta_{i}}\right]= \\
& =\frac{1}{\mu_{i+1}}\left[\left(\frac{\partial A_{i+1}}{\partial r}\right)_{r=R_{i}}+M_{\theta_{i+1}}\right]
\end{aligned}
$$

avec :

$$
\left\{\begin{array}{l}
M_{\theta_{1}}=M_{\theta} ; \quad M_{\theta_{2}}=M_{\theta_{3}}=0 \\
\mu_{1}=\mu_{0} \quad\left(\text { ou } \mu_{p} \text { si les aimants sont jointifs) } ;\right. \\
\mu_{2}=\mu_{3}=\mu_{0} .
\end{array}\right.
$$

En portant enfin les expressions (5), (6) et (7) du potentiel vecteur dans les relations (8) et (9), on obtient un système de 6 équations permettant de déterminer aisément les 6 inconnues $C_{g}, D_{g}, E_{g}$, $F_{g}, G_{g}, H_{g}$, dont les expressions sont données en annexe.

Notons que les relations (8) et (9) font intervenir seulement la composante tangentielle de l'aimantation.

calcul est donc différente suivant le type de leur aimantation.

En effet :

- Si $M_{\theta}=0$ (aimantation radiale), les aimants interviennent dans le calcul par le $2^{\mathrm{e}}$ membre de l'équation (2) $\left(\frac{\partial M_{r}}{\partial \theta}\right)$ qui est nul partout sauf sur les faces latérales des aimants. Dans ce cas la présence des aimants n'introduit pas de discontinuité aux limites entre zones.

- $\mathrm{Si} \quad M_{\theta} \neq 0$ (aimantation quelconque), les 
aimants interviennent à la fois par le second membre de l'équation (2) et par la discontinuité qu'ils introduisent sur les limites entre zones par le terme $M_{\theta}$.

En particulier si l'aimantation est telle que $M_{r}=M \cos \theta$ et $M_{\theta}=-M \sin \theta \quad(M$ parallèle à l'axe $\theta=0$ ), l'introduction des aimants dans le calcul ne se fait que par les conditions aux limites (le second membre de l'Eq. (2) est en effet alors nul).

\section{Détermination des grandeurs caractéristiques et validation.}

La connaissance du potentiel vecteur dans les différentes zones du domaine d'étude permet d'accéder aux grandeurs électromagnétiques caractérisant le fonctionnement de la machine, telles que inductions, flux, couple...

\subsection{EXPRESSION DES DIFFÉRENTES GRANDEURS.}

3.1.1 Calcul des inductions radiale et tangentielle. Les inductions radiale $B_{r}$ et tangentielle $B_{\theta}$ ont pour expression en fonction du potentiel vecteur:

$$
B_{r}=\frac{1}{r} \frac{\partial A}{\partial \theta}, \quad B_{\theta}=-\frac{\partial A}{\partial r} .
$$

Ainsi, par simple dérivation des relations (5), (6), (7), on peut calculer les inductions radiale et tangentielle dans les trois zones du domaine.

Nous donnons ci-dessous les expressions correspondant à la zone 3 de l'induit. La connaissance de l'induction dans cette zone est essentielle dans la mesure où elle intervient directement dans la création des forces de Laplace s'exerçant sur les conducteurs.

Il vient :

$$
\begin{aligned}
& B_{r}(r, \theta, t)=2 \sum_{g=1}^{+\infty} \mathcal{R e}\left[j g p\left(G_{g} r^{g p-1}+H_{g} r^{-g p-1}+\frac{\beta_{g}(r)}{r}\right) \mathrm{e}^{j g p \theta}\right] \\
& B_{\theta}(r, \theta, t)=-2 \sum_{g=1}^{+\infty} \operatorname{Re}\left[\left(g p G_{g} r^{g p-1}-g p H_{g} r^{-g p-1}+\frac{\partial \beta_{g}}{\partial r}\right) \mathrm{e}^{j g p \theta}\right] .
\end{aligned}
$$

3.1.2 Calcul de flux et de f.e.m. - Considérons un bobinage de la machine, décrit par la fonction densité de conducteurs $C(\theta)$, à travers lequel on désire calculer le flux.

Si $\Phi_{0}$ représente le flux embrassé par une spire (spo) de ce bobinage, dont la surface $\left(S_{0}\right)$ est délimitée par le contour $\left(\mathrm{b}_{0}\right)$, on $\mathrm{a}$ :

$$
\Phi_{0}=\iint_{\left(\mathbf{S}_{0}\right)} \mathbf{B} \cdot \mathrm{d} \mathbf{S}=\iint_{\left(\mathbf{S}_{0}\right)} \operatorname{rot} \mathbf{A} \cdot \mathrm{d} \mathbf{S}=\int_{\left(\mathrm{b}_{0}\right)} \mathbf{A} \cdot \mathrm{d} \mathbf{l} .
$$

Soient $(r, \theta)$ et $\left(r^{\prime}, \theta^{\prime}\right)$ les coordonnées des positions du conducteur "aller» et du conducteur "retour» de (spo). Compte tenu de l'invariance par translation suivant $\mathrm{O} z$ du système, la relation précédente devient :

$$
\Phi_{0}=A_{3}(r, \theta, t) . L-A_{3}\left(r^{\prime}, \theta^{\prime}, t\right) \cdot L
$$

où $L$ représente la longueur de la machine.

Dès lors, si l'on s'intéresse au flux élémentaire embrassé par un ensemble de spires infiniment voisines de la spire (spo), formant, dans le plan de la figure, une section de conducteurs ds, il vient :

$$
\begin{array}{r}
\mathrm{d} \Phi=\left(A_{3}(r, \theta, t) \cdot L-A_{3}\left(r^{\prime}, \theta^{\prime}, t\right) \cdot L\right) \times \\
\times C(\theta) \mathrm{d} s .
\end{array}
$$

En remarquant en outre qu'il existe une symétrie entre les groupements de conducteurs "aller» et "retour», on a :

$$
C(\theta)=-C\left(\theta^{\prime}\right) .
$$

Il est alors possible d'écrire :

$$
\begin{aligned}
\mathrm{d} \dot{\Phi}=A_{3}(r, \theta, t) & C(\theta) \cdot L \cdot \mathrm{d} s+ \\
& +A_{3}\left(r^{\prime}, \theta^{\prime}, t\right) \cdot C\left(\theta^{\prime}\right) \cdot L \cdot \mathrm{d} s .
\end{aligned}
$$

Par conséquent, en étendant le calcul à l'ensemble des spires constituant le bobinage considéré, on obtient, si $(\mathbf{S})$ et $\left(\mathrm{S}^{\prime}\right)$ représentent les domaines formés respectivement par les groupements de conducteurs «aller» et « retour» :

$$
\Phi(t)=L \cdot \iint_{(\mathrm{S})+\left(\mathrm{S}^{\prime}\right)} A_{3}(r, \theta, t) \cdot C(\theta) \cdot \mathrm{d} s
$$

la fonction $C(\theta)$ étant nulle dans toutes les parties de la zone des courants (zone 3) non occupée par le bobinage considéré, on peut écrire finalement :

$$
\Phi(t)=L \cdot \int_{R_{2}}^{R_{3}} \int_{0}^{2 \pi} A_{3}(r, \theta, t) \cdot C(\theta) r \mathrm{~d} \theta \mathrm{d} r .
$$

Si on désire par exemple évaluer le flux à travers la 
phase 1 , dont la densité de conducteurs, déterminée au paragraphe 1.2.1, peut s'écrire sous la forme :

$$
C_{1}(\theta)=\sum_{g=-\infty}^{+\infty} \mathrm{S}_{g} \mathrm{e}^{j g p \theta}
$$

les calculs conduisent aux relations suivantes :

$$
\Phi(t)=\sum_{g=1}^{+\infty} 4 \pi L\left(Y_{g_{1}}+Y_{g_{2}}+Y_{g_{3}}\right)
$$

avec :

$$
\begin{aligned}
& Y_{g_{1}}=\frac{\mathcal{R e}\left[\mathrm{S}_{g} \cdot G_{g}^{*}\right]}{g p+2}\left(R_{3}^{g p+2}-R_{2}^{g p+2}\right) \\
& Y_{g_{2}}=\operatorname{Re}\left[\mathrm{S}_{g} \cdot H_{g}^{*}\right] \cdot \log \frac{R_{3}}{R_{2}} \text { si } g p=2 \\
& Y_{g_{2}}=\frac{\operatorname{Re}\left[\mathrm{S}_{g} \cdot H_{g}^{*}\right]}{2-g p}\left(R_{3}^{2-g p}-R_{2}^{2-g p}\right) \text { si } g p \neq 2 \\
& Y_{g_{3}}=\left[\frac{\operatorname{Re}\left[\mathcal{S}_{g} \cdot \beta_{g}^{*}(r)\right]}{16} r^{2}\left(4-\frac{1}{\log r}\right)\right]_{R_{2}}^{R_{3}}
\end{aligned}
$$

$Y_{g_{3}}=\left[\frac{\operatorname{Re}\left[\mathcal{S}_{g} \cdot \beta_{g}^{*}(r)\right]}{4} r^{2}\right]_{R_{2}}^{R_{3}} \operatorname{sig} g \neq 2$.

A partir de la connaissance du flux, on peut déduire, en dérivant par rapport au temps, la f.e.m. aux bornes du bobinage considéré.

3.1.3 Couple électromagnétique. - Le couple électromagnétique est calculé à partir du tenseur de Maxwell [13]. Pour cela, nous utilisons une surface $S$ cylindrique, de rayon $R$, située dans l'entrefer (zone 2) et entourant le rotor. Le moment par rapport à l'axe de la machine $(\mathrm{O} z)$ de l'ensemble des forces qui s'exercent sur le rotor s'exprime alors par :

$$
\mathbf{k} \cdot \mathrm{C}=\int_{(\mathrm{S})} R(\mathbf{T} \wedge \mathrm{d} \mathbf{S})
$$

avec :

$$
\mathbf{T}=\mu_{0}\left[(\mathbf{n} \cdot \mathbf{H}) \mathbf{H}-\frac{\mathbf{n}}{2}(\mathbf{H})^{2}\right]
$$

où $\mathbf{k}$ et $\mathbf{n}$ désignent respectivement le vecteur unitaire suivant l'axe $\mathrm{O} z$ et la normale à la surface d'intégration $\mathrm{S}$.

On en déduit après calcul l'expression du couple délivré par la machine:

$$
\mathcal{C}(t)=R^{2} L \int_{0}^{2 \pi} \mu_{0} H_{r}(R, \theta, t) . H_{\theta}(R, \theta, t) \mathrm{d} \theta
$$

On a donc en fonction du potentiel vecteur dans l'entrefer :

$$
\mathcal{C}(t)=-\frac{R L}{\mu_{0}} \int_{0}^{2 \pi}\left(\frac{\partial A_{2}}{\partial \theta}\right)_{r=R} \cdot\left(\frac{\partial A_{2}}{\partial r}\right)_{r=R} \mathrm{~d} \theta .
$$

En utilisant l'expression (5) définissant le potentiel vecteur dans cette zone, on obtient après calcul :

$$
\mathcal{C}(t)=\frac{8 \pi p^{2} L}{\mu_{0}} \sum_{g=1}^{+\infty} g^{2} \operatorname{Im}\left[E_{g}^{*}(t) \cdot F_{g}(t)\right]
$$

Notons que le couple peut aussi se calculer directement en effectuant la sommation sur l'ensemble du bobinage des forces de Laplace s'exerçant sur les conducteurs :

Si $B_{r_{\mathrm{V}}}(r, \theta, t)$ désigne l'induction radiale à vide dans la zone (3), calculée à l'aide de la relation (10), on a :

$\mathcal{C}(t)=\int_{R_{2}}^{R_{3}} \int_{0}^{2 \pi} L \cdot B_{r_{\mathrm{V}}}(r, \theta, t) . J(\theta, t) \cdot r^{2} \mathrm{~d} \theta \mathrm{d} r$.

Cette deuxième méthode conduit au même résultat que le calcul par le tenseur de Maxwell.

3.2 RÉSultats et validation. - Compte tenu de la relative complexité des expressions analytiques obtenues, nous avons développé un logiciel, qui à partir des données que constituent les dimensions de la machine, les caractéristiques de l'inducteur et de l'induit et les conditions de fonctionnement, permet d'observer l'évolution des diverses grandeurs électromagnétiques.

En vue de valider la modélisation, nous avons procédé à la confrontation des résultats théoriques obtenus avec ceux issus de l'expérience sur un prototype de machine sans encoches à 3 paires de pôles réalisé au laboratoire. La structure et les dimensions principales sont données sur la figure 5 où est représenté un pas polaire.

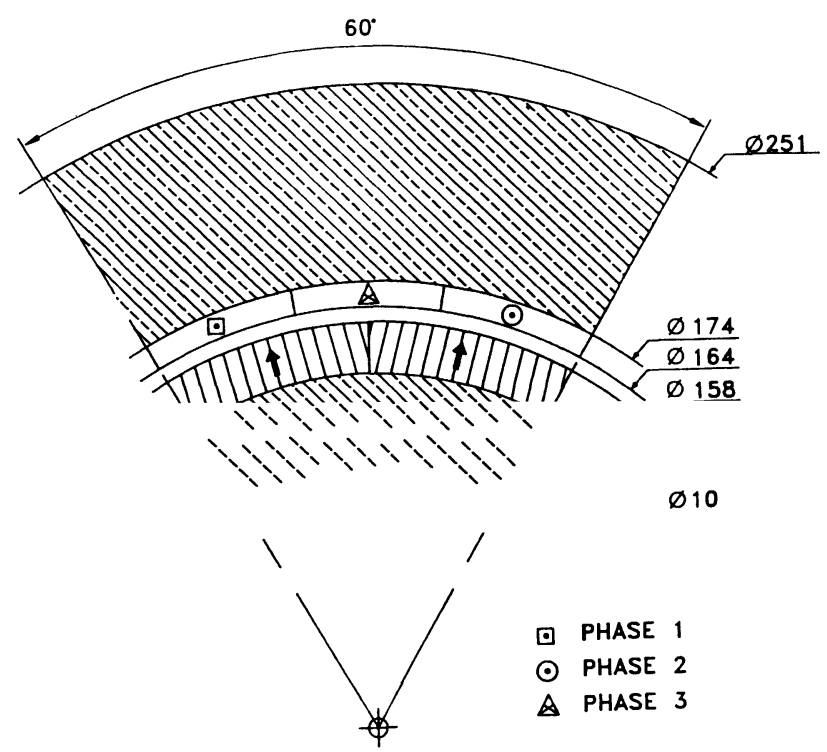

Fig. 5. - Caractéristiques du prototype (dimensions en $\mathrm{mm})$.

[Characteristics of the prototype (dimensions in $\mathrm{mm}$.] 
L'inducteur est réalisé à partir d'aimants Samarium Cobalt dont la valeur typique d'aimantation est de 0,9 T. Ces aimants sont taillés en forme de tuiles et assemblés jointirement. Chayue pôle ent réaline au moyen de deux blocs juxtaposés et aimantés parallèlement à leur axe de symétrie.

L'induit de la machine, dont le bobinage triphasé est a pals diamétral, forme une couche de $5 \mathrm{~mm}$
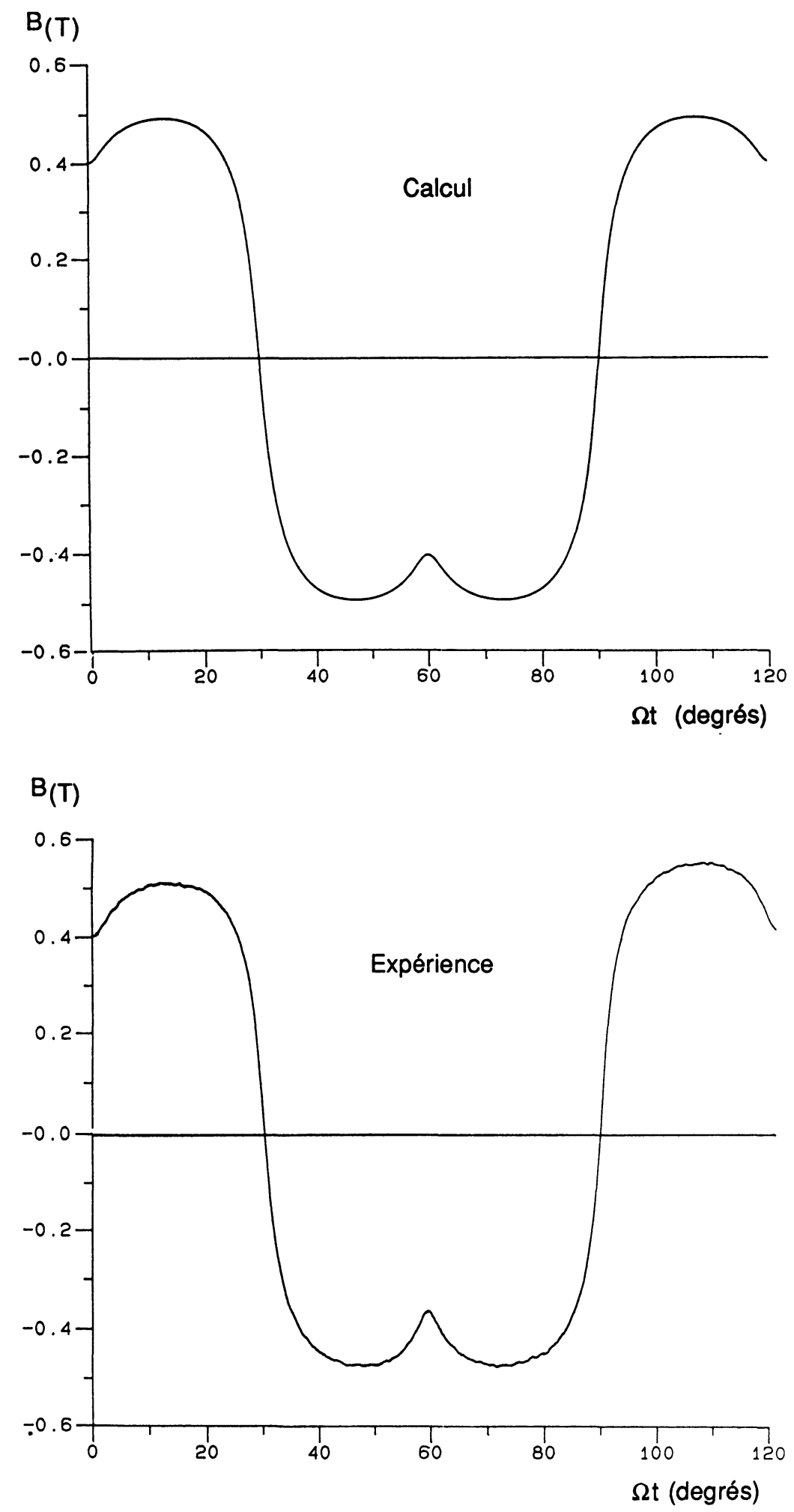

Fig. 6. - Induction à vide en un point à la surface de l'induit.

[No-load flux density at a point on the windings surface.] 
d'épaisseur dans laquelle les conducteurs, de diamètre $1 \mathrm{~mm}$, sont jointifs.

Les culasses du rotor et du stator sont largement dimensionnées pour éviter la saturation du matériau magnétique.
La longueur de la machine suivant son axe est de $40 \mathrm{~mm}$.

Notons que, pour l'exemple traité ici, les calculs de série ont été limités au rang $g=60$. En raison du contenu harmonique des formes d'ondes des gran-

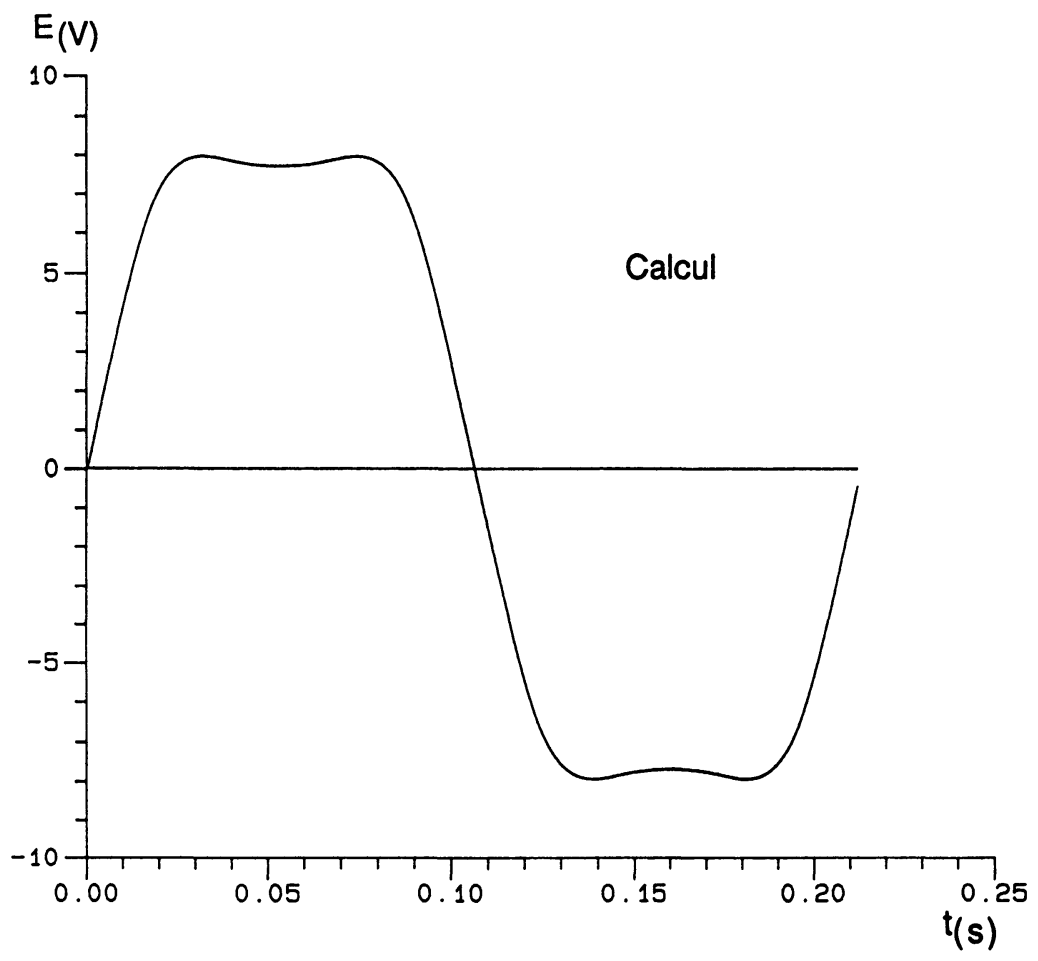

$E(V)$

10

Fig. 7. - F.e.m. aux bornes d'une phase.

[Phase emf.] 
deurs observées, cette valeur est largement suffisante pour assurer une convergence satisfaisante.

Nous donnons sur la figure 6 les courbes théoriques et expérimentales, représentant l'évolution de l'induction à vide en un point du bobinage. La forme particulière de cette courbe est une conséquence de la réalisation de l'inducteur au moyen de deux blocs aimantés parallèlement et juxtaposés.
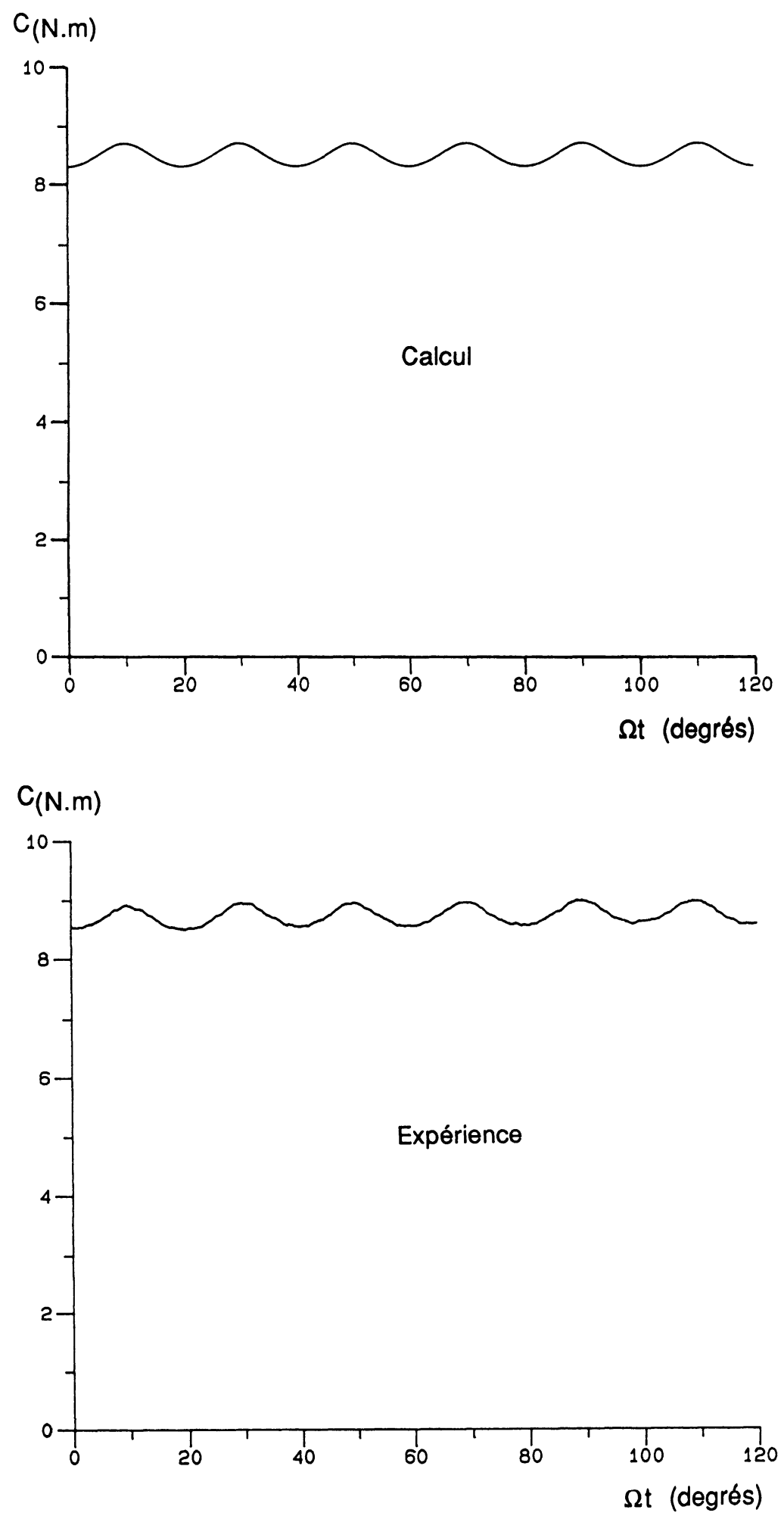

Fig. 8. - Couple avec une alimentation par des courants triphasés sinusoïdaux.

[Torque with a three-phased sinusoidal current supply.] 
Nous présentons sur la figure 7 les résultats concernant la f.e.m. aux bornes d'une phase du bobinage triphasé. Son allure est directement liée à la forme de l'induction délivrée par l'inducteur et à la répartition des conducteurs.

L'induit étant alimenté par un système triphasé de courants sinusoïdaux, nous donnons sur la figure 8 les relevés correspondant au couple électromagnétique développé par la machine. La répartition de l'induction n'ayant pas été spécialement optimisée dans le prototype, le couple obtenu présente des ondulations relativement importantes.

L'ensemble de ces résultats valide bien la méthode de modélisation. Au niveau qualitatif, on peut observer une très bonne concordance entre les formes d'ondes calculées et celles obtenues expérimentalement. Sur le plan quantitatif, les écarts sont très faibles, inférieurs aux marges d'incertitudes. On peut cependant remarquer que, dans ce type de structure de machine relativement simple, les principaux paramètres sensibles sont connus avec une bonne précision, sauf l'aimantation des aimants pour laquelle il existe une certaine dispersion. Pour le calcul nous avons adopté la valeur typique donnée par le constructeur $(0,9 \mathrm{~T})$ et les résultats de la figure 6 , relatifs à l'induction en un point du bobinage, ont permis de confirmer la validité de ce choix.

\section{Conclusion.}

Nous avons présenté, dans cet article, une méthode d'étude des machines à aimants sans encoches, mettant en œuvre un calcul analytique du champ en deux dimensions. Après en avoir exposé les bases, nous avons décrit la modélisation des sources du champ, conçue de façon à prendre en compte des structures d'inducteur et d'induit relativement élaborées. Nous avons ensuite établi les expressions analytiques du potentiel vecteur dans les différentes zones du domaine d'étude, ce qui nous a permis de déterminer les expressions des diverses grandeurs intervenant dans la machine. La confrontation des résultats théoriques avec ceux issus de l'expérience s'est enfin avérée très satisfaisante.

Cette modélisation paraît donc très bien adaptée à l'étude des machines à induit sans encoches. La méthode, en permettant d'accéder aux expressions des diverses grandeurs, fait apparaître directement l'influence des différents paramètres définissant la machine, ce qui rend l'outil développé particulièrement efficace dans le cadre d'une optimisation. Ainsi, il est actuellement utilisé pour étudier les conditions de minimisation des ondulations de couple de machine de forte puissance en examinant l'influence de différentes répartitions d'aimants et/ou de conducteurs.

\section{Bibliographie}

[1] DAvies E. J., Airgap winding for large turbogenerators, Proc. IEE, Vol. 118, n $3 / 4$ (1971).

[2] SPOONER E., Fully slotless turbogenerators, Proc. IEE, Vol. 120, $\mathrm{n}^{\circ} 12$ (1973).

[3] Watanabe M., Takahashi M., Takahashi N., TsuKui T., Experimental study for a practical winding stator arrangement for large turbine generators, IEEE Trans. Power Apparatus Syst. Vol. PAS-100, $n^{\circ} 4$ (1981).

[4] Bocharov V. I., Sedov V. I., Evaluating the therma oa ing o a s ot ess traction-motor armature, Elektrotekhnika, Vol. 47, n 12 (1976).

[5] SPOONER E., D. C. traction motor with slotless armature, Proc. IEE, Vol. 132, $\mathrm{n}^{\circ} 2$ (1985).

[6] GutT J. J., Schröder M., Brushless positioning drives with extremely low pulsation of the torque, ICEM, Munich, RFA (September 8-10, 1986).

[7] England T. R., Unique surface-wound brushless servo with improved torque ripple characteristics, IEEE Trans. Ind. Appl., Vol. 24, $\mathrm{n}^{\circ} 6$ (1988).

[8] Laporte B., Berenger C., Permanent magnet synchronous machines owning windings in the airgap, ICEM, Pisa, Italy (September 12-14, 1988).

[9] BANDelier B., Rioux-Damidau F., Modélisation par séries de Fourier de machines synchrones à aimants, Journées d'étude SEE, Développements récents des méthodes numériques appliquées aux machines électriques, 13 mars 1987, Gif-sur-Yvette.

[10] Boules N., Two dimensional field analysis of cylindrical machines with permanent magnet excitation, IEEE Trans. Ind. Appl., Vol. IA-20, $\mathrm{n}^{\circ} 5$ (1984).

[11] Yoshida K., WeH H., Méthode de simulation d'aimants permanents en vue d'un traitement analytique des machines électriques, Arch. electrotech., $\mathrm{n}^{\circ} 68$ (1985).

[12] Eid G., Mouillet A., D. C. brushless motors with a rare earth permanent magnet rotor, Zeszyty Naukowe Politechniki Poznanskiej Elektryka, Poznan, $n^{\circ}$ 21, 1980.

[13] Durand E., Magnétostatique (Masson et Cie Editeurs) 1968. 
720

REVUE DE PHYSIQUE APPLIQUÉE

N 7

Annexe

Expression des constantes définissant le potentiel vecteur.

En posant :

$\left\{\begin{array}{l}\alpha_{g}^{\prime}\left(R_{i}\right)=\left(\frac{\partial \alpha_{g}}{\partial r}\right)_{r=R_{i}}+\mathcal{M}_{\theta_{g}} \\ \beta_{g}^{\prime}\left(R_{i}\right)=\left(\frac{\partial \beta_{g}}{\partial r}\right)_{r=R_{i}} .\end{array}\right.$

On obtient :

$$
\begin{aligned}
C_{g}= & \frac{1}{R_{3}^{g p}-R_{3}^{-g p} R_{0}^{2 g} p}\left[\frac{R_{3}^{-g p} R_{0}^{g p+1}}{g p} \alpha_{g}^{\prime}\left(R_{0}\right)-\frac{R_{3}}{g p} \beta_{g}^{\prime}\left(R_{3}\right)-\frac{R_{3}^{g p} R_{1}^{-g p}-R_{3}^{-g p} R_{1}^{g p}}{2} \alpha_{g}\left(R_{1}\right)-\right. \\
& \left.-\frac{R_{3}^{g p} R_{1}^{1-g p}+R_{3}^{-g p} R_{1}^{g p+1}}{2 g p} \alpha_{g}^{\prime}\left(R_{1}\right)+\frac{R_{3}^{g p} R_{2}^{-g p}-R_{3}^{-g p} R_{2}^{g p}}{2} \beta_{g}\left(R_{2}\right)+\frac{\left.R_{3}^{g p} R_{2}^{1-g p}+R_{3}^{-g p} R_{2}^{g p+1} \beta_{g}^{\prime}\left(R_{2}\right)\right]}{2 g p}\right) \\
D_{g}= & C_{g} R_{0}^{2 g p}+\frac{R_{0}^{g p+1}}{g p} \alpha_{g}^{\prime}\left(R_{0}\right) \\
E_{g}= & C_{g}+\frac{R_{1}^{-g p}}{2} \alpha_{g}\left(R_{1}\right)+\frac{R_{1}^{1-g p}}{2 g p} \alpha_{g}^{\prime}\left(R_{1}\right) \\
F_{g}= & C_{g} R_{0}^{2 g p}+\frac{R_{0}^{g p+1}}{g p} \alpha_{g}^{\prime}\left(R_{0}\right)+\frac{R_{1}^{g p}}{2} \alpha_{g}\left(R_{1}\right)-\frac{R_{1}^{g p+1}}{2 g p} \alpha_{g}^{\prime}\left(R_{1}\right) \\
G_{g}= & C_{g}+\frac{R_{1}^{-g p}}{2} \alpha_{g}\left(R_{1}\right)+\frac{R_{1}^{1-g p}}{2 g p} \alpha_{g}^{\prime}\left(R_{1}\right)-\frac{R_{2}^{-g p}}{2} \beta_{g}\left(R_{2}\right)-\frac{R_{2}^{1-g p}}{2 g p} \beta_{g}^{\prime}\left(R_{2}\right) \\
H_{g}= & C_{g} R_{0}^{2 g p}+\frac{R_{0}^{g p+1}}{g p} \alpha_{g}^{\prime}\left(R_{0}\right)+\frac{R_{1}^{g p}}{2} \alpha_{g}\left(R_{1}\right)-\frac{R_{1}^{g p+1}}{2 g p} \alpha_{g}^{\prime}\left(R_{1}\right)-\frac{R_{2}^{g p}}{2} \beta_{g}\left(R_{2}\right)+\frac{R_{2}^{g p+1}}{2 g p} \beta_{g}^{\prime}\left(R_{2}\right) .
\end{aligned}
$$

